\title{
Génétique des populations de chats domestiques de l'île de la Réunion
}

\author{
P Dreux \\ ENS, laboratoire de zoologie, 46, rue d'Ulm, 75230 Paris cedex 05, France
}

(Reçu le 20 mars 1990; accepté le 13 juin 1990)

\begin{abstract}
Résumé - La population des chats domestiques de la Réunion a été examinée, particulièrement dans la ville principale, Saint-Denis. L'application du test de Hardy-Weinberg au gène orange a montré qu'elle était panmictique. La fréquence des allèles révèle que cette population de chats a pour origine des individus venant de France et d'autres venant principalement d'Asie, à la suite du peuplement humain.
\end{abstract}

chat / génétique / île de la Réunion / population

Summary - Cat population genetics of Reunion Island. A survey of the domestic cat population of Reunion Island, chiefly in the principal town, Saint-Denis, has been performed. The Hardy-Weinberg test at the Orange gene shows it to be a randomly breeding population. Gene frequencies suggest a double origin of cats, ie from France and from several regions in Asia, following human settlement.

domestic cat / genetics / Reunion Island / population

\section{INTRODUCTION}

Depuis plus de 20 ans, de brefs séjours à l'île de la Réunion, avant ou après des missions aux Terres australes françaises, ont permis d'examiner la population de chats, l'investigation de beaucoup la plus importante (plus de la moitié de l'effectif) ayant eu lieu en janvier 1989. Ces différents sondages en milieu urbain ont eu lieu principalement à St.Denis, la ville la plus importante de l'île, et ont permis de connaître la fréquence des allèles mutants aux différents loci codant principalement pour la couleur du pelage. Quelques chats ont été examinés en dehors des agglomérations, mais leur nombre est très faible et ils ont été réunis pour ce travail à tous ceux recensés à St-Denis et à ceux, beaucoup moins nombreux, recensés dans la localité urbaine du Port. Il importe de noter que ces villes ne présentent pas un caractère urbain très prononcé; les jardins, les terrains vagues y sont fréquents. Un sondage préliminaire avait déjà été publié (Dreux, 1969) portant seulement sur 18 individus, et l'étude présente, portant sur 256 individus, est évidemment beaucoup plus fiable; elle présente d'ailleurs de grandes différences avec celle de 1969, non pour la fréquence des allèles communs qui est presque la 
même que celle de ce sondage très réduit, mais parce qu'il montre la présence d'allèles plus rares.

Le recensement a été fait presque exclusivement en parcourant les différentes parties de l'agglomération de St-Denis et en notant tout chat rencontré, principalement aux heures où les chats sont les plus visibles, c'est-à-dire dans les brèves périodes de l'aube et du crépuscule. Un quadrillage soigné du plan de St-Denis et une notation précise des chats rencontrés permettaient de ne pas noter un individu plusieurs fois. Le refuge de la Société protectrice des animaux n'héberge que des chiens; il n'a donc pas pu être utilisé pour ce sondage. Une enquête auprès des vétérinaires n'aurait d'autre part été que d'un faible rendement.

\section{RÉSULTATS GÉNÉRAUX}

Sur les 256 chats recensés, 72 ont pu être reconnus comme mâles et 72 comme femelles, le sexe des 112 restants n'ayant pu être déterminé. On admettra donc dans la suite que la sex-ratio est de 1 . Cinquante-cinq étaient des jeunes, proportion trop faible pour pouvoir les comparer efficacement aux adultes. Il n'a pu être déterminé si certains de ces chats étaient castrés ou stérilisés, mais leur nombre doit être très faible.

Dix d'entre eux avaient la queue plus ou moins tordue (gène $K t$ présumé dominant) (Searle, 1959), et un, les oreilles pliées (gène $\boldsymbol{F d}$, dominant) (Robinson, 1975). En général, il n'a pas été possible d'examiner les animaux d'assez près pour voir s'il y en avait de polydactyles ou de syndactyles, et aucun n'a été reconnu.

\section{Gène orange; panmixie}

Cinq chats étaient porteurs du gène $W^{+}$(blanc dominant), et il en restait donc 251 classifiables pour le gène orange $O$, semi-dominant. Les 43 individus de phénotype écaille de tortue ont été présumés tous femelles et un effort d'approche particulier a été fait pour les phénotypes orange, permettant de déterminer le sexe de tous avec suffisamment de sécurité: 34 mâles et 5 femelles. Ceci a permis de faire le test classique de panmixie sur le gène orange, en admettant que les individus classifiables se répartissaient en 126 mâles et 125 femelles (tableau I).

Tableau I. Distributions observées et calculées (entre parenthèses) des phénotypes correspondant au gène orange dans la population de chats domestiques de l'île de la Réunion.

\begin{tabular}{lcll}
\hline Génotypes & $0 /-$ ou $0 / 0$ & $0 /+$ & $+/-$ ou $+/+$ \\
\hline Mâles (126) & $34(29)$ & - & $92(97)$ \\
Femelles (125) & $5(6,6)$ & $43(44,3)$ & $77(74)$ \\
\hline
\end{tabular}

Le $\chi^{2}$ est de 2,75 pour 3 ddl et, même au seuil de probabilité de $10 \%$, il n'y a pas de raison de douter de la panmixie de la population pour le gène $O$. 


\section{Fréquences géniques}

En supposant que la population de St-Denis était également panmictique pour les autres loci, on a calculé la fréquence de leurs allèles mutants. Ces fréquences sont données dans le tableau II où figure aussi celle de $O$. Les effectifs phénotypiques qui y sont mentionnés sont ceux de l'homozygote pour les allèles récessifs (exemple : $a a$ ), ceux de l'homozygote et de l'hétérozygote pour les allèles dominants (exemple: $\left.W^{+}\right)$. L'intervalle de confiance avec une probabilité de $95 \%$ a été donné dans la dernière colonne, la distribution pour les gènes rares n'étant pas symétrique autour de la moyenne. De plus, 4 chats avaient le phénotype chinchilla, tous en présence du gène agouti $A$; il semble que ce phénotype puisse être dû à 2 gènes différents, aussi une estimation de fréquence n'a-t-elle pas été faite.

Tableau II. Fréquences des gènes dans la population de chats domestiques de l'île de la Réunion.

\begin{tabular}{llllll}
\hline Allèles & $\begin{array}{l}\text { Nombres } \\
\text { classifiables }\end{array}$ & $\begin{array}{l}\text { Effectifs } \\
\text { phénotypiques }\end{array}$ & $\begin{array}{l}\text { Fréquences } \\
\text { phénotypiques }\end{array}$ & $\begin{array}{l}\text { Fréquences } \\
\text { géniques }\end{array}$ & $\begin{array}{l}\text { Intervalle de } \\
\text { confiance } \dot{a} 95 \%\end{array}$ \\
\hline$a$ & 212 & 116 & 0,55 & 0,74 & $0,69-0,78$ \\
$c^{s}$ & 251 & 15 & 0,06 & 0,24 & $0,19-0,31$ \\
$d$ & 251 & 22 & 0,088 & 0,30 & $0,24-0,36$ \\
$l$ & 256 & 8 & 0,031 & 0,17 & $0,10-0,24$ \\
$O$ & 251 & - & - & 0,23 & $0,19-0,28$ \\
$S$ & 251 & 118 & 0,47 & - & - \\
$T^{a}$ & 126 & 17 & 0,136 & 0,07 & $0,04-0,11$ \\
$t^{+}$ & 126 & 97 & 0,770 & 0,62 & $0,56-0,68$ \\
$t^{b}$ & 126 & 12 & 0,095 & 0,31 & $0,23-0,40$ \\
$W$ & 256 & 5 & 0,019 & 0,01 & - \\
\hline
\end{tabular}

\section{Panachure}

Tous les chats qui ne présentaient pas le phénotype blanc dominant $W^{+}$, soit 251 , ont été classés dans l'échelle de panachure précédemment définie (Dreux, 1967, 1968,1987 ) en catégories de 0 à 0,9 , les nombres exprimant approximativement la fraction du corps de couleur blanche (tableau III); la catégorie $\varepsilon$ indique la présence sous la gorge ou sous le ventre, ou les deux, d'une petite touffe de poils blancs. Des recherches en cours semblent montrer que le déterminisme de cette panachure est moins simple qu'on ne le pensait, nous n'avons donc pas fait d'estimation de fréquence génique.

Tableau III. Classes de répartition de la panachure dans la population de chats domestiques de l'île de la Réunion.

\begin{tabular}{llllllllllll}
\hline Classe de panachure & 0 & $\varepsilon$ & 1 & 2 & 3 & 4 & 5 & 6 & 7 & 8 & 9 \\
Nombres & 126 & 7 & 23 & 25 & 22 & 15 & 10 & 5 & 3 & 6 & 9 \\
\hline
\end{tabular}




\section{DISCUSSION ET CONCLUSION}

On peut considérer que dans une île comme La Réunion, le peuplement de chats est un reflet approximatif de l'immigration humaine qui y a apporté ses animaux domestiques. Il est donc normal d'y retrouver une certaine proportion d'allèles $t^{b}$, particulièrement abondants en Europe occidentale, et qui révèle la colonisation française; mais leur fréquence est beaucoup plus faible qu'en France.

On est donc amené à penser qu'une forte immigration hindoue et chinoise a apporté à La Réunion des traits caractéristiques des populations de chats d'Extrême-Orient: fréquence assez forte de $O$ et de $T^{a}$. L'allèle siamois $c^{s}$ est assez abondant, mais finalement guère plus qu'en Europe occidentale où il se répand rapidement, favorisé par les goûts des propriétaires (Dreux, 1986). On trouvera dans le tableau IV une comparaison, nullement exhaustive à cause du grand nombre d'études déjà publiées, de la population de chats de La Réunion avec celles de quelques localités caractéristiques, et qui met en évidence son caractère composite.

Tableau IV. Fréquences des gènes dans quelques populations de chats domestiques en France et dans l'océan Indien.

\begin{tabular}{lllllll}
\hline Allèles & $a$ & $O$ & $c^{s}$ & $T^{a}$ & $t^{+}$ & $t^{b}$ \\
\hline France: & & & & & & \\
$\quad$ Paris & 0,71 & 0,06 & 0,03 & 0,001 & 0,22 & 0,78 \\
$\quad$ moyenne & 0,70 & 0,11 & variable & 0 & 0,35 & 0,65 \\
Afrique du Sud & 0,79 & 0,18 & nul? & 0,01 & 0,41 & 0,58 \\
Singapour & 0,60 & 0,31 & 0,13 & 0,24 & 0,65 & 0,11 \\
Pakistan & 0,58 & 0,26 & - & 0,01 & 0,85 & 0,14 \\
Bangkok & 0,72 & 0,30 & 0,29 & 0,05 & 0,95 & 0 \\
Seychelles & 0,67 & 0,26 & 0 & 0,07 & 0,93 & 0 \\
Réunion (1969) & 0,78 & 0,25 & 0,24 & 0,05 & 0,63 & 0,32 \\
Réunion (1989) & 0,74 & 0,23 & 0,24 & 0,07 & 0,62 & 0,31 \\
\hline
\end{tabular}

Les résultats publiés en 1969 ont été rappelés dans ce même tableau; leur valeur statistique, portant seulement sur 18 individus, est très faible. Cependant, leur étroite similitude avec les fréquences données dans cet article est un argument en faveur de la stabilité actuelle, depuis 20 ans, du pool génique de la population.

\section{RÉFÉRENCES}

Dreux $\mathrm{P}$ (1967) Gene frequencies in the cat population of Paris. $J$ Hered, 58, 89-92 Dreux P (1968) Panachure chez le chat (II). Carniv Genet Newsl I (4), 64-66

Dreux P (1969) The cats of Kerguelen Island (South Indian Ocean), Réunion and Bloemfontein (Orange Free State, South Africa). Carniv Genet Newsl I (7), 170

Dreux $\mathrm{P}$ (1986) Génétique des populations de chats domestiques dans un district de Bretagne (Carnac, Morbihan, France). Colloque National CNRS: Biologie des populations, Lyon, 4-6 sept 1986, 312-316 
Dreux P (1987) Déterminisme génétique de la couleur de la robe chez le chat domestique. Ethnozootechnie, 40s, s71-s79

Robinson R (1975) Further data on the folded eard cats. Carniv Genet Newsl II (10), 326-327

Searle AG (1959) A study of variation in Singapore cats. J Gen 56, 1-16 